\title{
Nonlinear Response of Cantilever Beams Due to Large Geometric Deformations: Experimental Validation
}

\author{
Claudia Aide Gonzalez-Cruz* - Juan Carlos Jauregui-Correa - Gilberto Herrera-Ruíz \\ Autonomous University of Querétaro, Faculty of Engineering, Mexico
}

Many structural elements, such as gas turbine fans, wind turbine blades, springboards among others are designed as slender elements. Since their dynamic behaviour can be modeled as cantilever beams, it is important to understand their nonlinear behaviour due to large deformations. In this way, this work presents the experimental validation of a simplified model of a cantilever beam. The model is formulated considering large geometric deformations and assuming a Galerkin approach. The model is validated experimentally, and it is found that there is a characteristic frequency related to the nonlinear terms. The data is analysed using time-frequency maps produced with the continuous wavelet transform.

Keywords: phase diagram, harmonic distortion, continuous wavelet transform, large geometric deformations

Highlights

- Large geometric deformations in cantilever beams produce nonlinear behaviour.

- The dynamic response of a cantilever beam is highly dominated by the structural damping.

- Cantilever beam is modeled with a nonlinear restoring force.

- Nonlinear frequencies only can be found numerically or experimentally.

\section{INTRODUCTION}

This paper presents an experimental validation of the nonlinear behaviour of cantilever beams. It is postulated that the nonlinear behaviour of a cantilever beam is due to large deformations. In order to validate this assumption, it is necessary to derive the nonlinear equations and compare them numerically with experimental results. From the experimental data, it is found that the dynamic response is highly dominated by the structural damping. This parameter diminishes the amplitude and, as a consequence, the nonlinear effect.

The subject has been studied for many years, and several researchers have proposed dynamic models and experimental procedures. Nevertheless, unanswered questions remain, and the validation has not been fulfilled. The bending of beams has been analysed especially for elastic problems. Halilovič and Štok [1] presented a deflection analysis of rectangular closed section beams with no hardening; assuming elastoplastic behavior, they derived analytical solutions considering small strains and small displacements. $\mathrm{Yu}$ et al. [2] modelled a naturally curved and twisted rectangular beam; their analysis considered a closed thin-walled section. The model was based on the principle of minimum potential energy. Although their model can be extended to solid cross-section beams, it is based on the small displacement theory. Regarding a large deformation model, Gerstmayr and Irschik [3] represented the large deformation using the elastic line approach. They used a cubic polynomial to represent the displacement; a similar approach is also applied in the present work. They used the weak form of the equation of motion using Lagrange's equation and D'Alembert's principle. Their results showed the vertical displacement of a beam as a function of time, and they never presented frequency or phase diagram analysis. Ashour and Nayfeh [4] presented a research on the nonlinear behaviour of an absorber to control vibrations in plates. The absorber is based on the saturation phenomenon associated with a quadratic nonlinear term and internal resonances. They identified two main problems regarding the application of auto-parametric absorbers: coupling two mechanical systems together and maintaining two-to-one internal resonances. For solving this problem, they applied a piezoelectric ceramic actuator; in their solution, they applied their method to a cantilever beam. Their beam model is only linear; the nonlinearity is in the actuator function. They found the solution applying Nayfeh's perturbation method.

The conventional method for the experimental study of nonlinear mechanical systems is parameter sweeping, in which the parameter is ramped smoothly up and down while the system response is recorded. This method is frequently used to demonstrate the bifurcation condition. Using a pathfollowing algorithm, Bureau et al. [5] developed a stabilizing control scheme for an experimental bifurcation analysis. In order to produce bifurcation, they proposed the application of a nonlinear 
electromagnetic actuator and a proportional-derivative (PD) controller. With this method, it was possible to trace the bifurcation path. In their results, the phase diagram lacked representation of the two attracting poles of a typical bifurcation solution. Malatkar and Nayfeh [6] described a procedure for determining the nonlinear parameters of a cantilever beam excited at the supported end. The bifurcation plot was constructed using the sweep technique. They found that the large deformation component was neglected in their experiments. Therefore, it is confirmed that damping suppresses the large deformation effect. Paak et al. [7] analysed the nonlinear vibrations of a cantilevered cylinder. In their model, they considered the large displacement terms in the strain formula and they approximated the displacements as sinusoidal series. Their results are similar to the phase diagrams presented in the following sections. In a similar work, Stoykov and Ribeiro [8] presented a nonlinear forced vibration model for beams. They found similar phase diagrams with high amplitudes in the excitation force. $\mathrm{Li}$ and Zhang [9] used the B-spline function to derive a dynamic model of a tapered beam. They approximated the deformation variables as a cubic B-spline function. Several researchers described different approaches for determining the nonlinear term. Stangl et al. [10] modelled a cantilevered pipe as a Euler elastic; they considered large deflections without order-ofmagnitude assumptions. Equations of motion were obtained using an extended version of the Lagrange equations in combination with a Ritz method. Wang and Liew [11] modelled elastodynamic problems using an improved complex variable moving leastsquare approximation and the Galerkin procedure. Furthermore, they enhanced the computing efficiency using an improved complex variable element-free method. Wang et al. [12] proposed a nonlinear model for a Euler-Bernoulli microscale beam. The governing equations are developed from the Hamilton principle following the modified couple stress theory and the Von Kármán geometrically nonlinear theory. The governing equations are reduced the partial differential governing equations to a nonlinear twopoint boundary value problem in a spatial variable by means of the Kantorovich time-averaging method. Zhang et al. [13] modelled a deploying-and-retreating wing as a cantilever laminated composite beam. They derived the governing equations of motions from the Hamilton's principle and Reddy's third-order theory and von Karman type equations of large deformation. By means of the Galerkin method, the dimensionless partial differential equation is transformed into a set of the ordinary differential equations. Noor et al. [14] developed simple mixed finite element models for curved thin-walled beams, the models include the effects of flexural-torsional coupling, the additional effects of transverse shear deformation, and rotary inertia. In general, it is assumed that large deflections are related to a nonlinear stiffness, whereas the damping term is always linear. Machado and Cortínez [15] developed a nonlinear model for beams. Their formulation only considered the elastic deformation, and they neglected the dynamic effects. Ramezani [16] derived a beam model based on the strain gradient elasticity theory. He assumed large deformations but proposed a dynamic equation with a stiffness function with a cubic coefficient. Kang and Li [17] presented a model of a cantilever beam with nonlinearities. They approximated the large deformations as a function of the stress-strain exponent. Fotouhi [18] presented a dynamic analysis of flexible beams. He considered only the free vibration case. He solved the problem using ANSYS. The results are presented in a way that is difficult to compare them with further analysis. He showed that large deformation analysis can be conducted with commercial software. Shad et al. [19] used a similar approach to the analysis of rotor dynamics using higher order deformations in bending. Although their model considers a simple supported beam, their analysis is similar to the analysis of cantilever beams. They showed bifurcations in the frequency spectrum.

The purpose of this paper is to demonstrate that the nonlinear behaviour of a cantilever beam is due to the large deformation term. Although the structural damping of the beam is a dominant parameter that diminishes large deformations, it is possible to reveal the nonlinear behaviour with the sweep method. Thus, in order to deploy the nonlinear terms, it is necessary to produce large deformations. Do so, in practice, is difficult to achieve.

The remainder of this paper is organized as follows. Section 1 presents the derivation of a simplified model using the beam deflection. Section 2 provides the analysis techniques used for data processing. Section 3 presents the numerical solution of the model for free vibration and forced vibration tests. Section 4 presents the experimental validation of the model. Finally, Section 5 gives the conclusions.

\section{DYNAMIC MODEL}

According to Gonzalez and Jauregui [20], nonlinear dynamic behaviour can be modelled using the beam deflection model. Since the purpose of this work is to demonstrate the effect of large deformations, the 
following development is based on the simplest beam model. As described by them, it is assumed that the material is homogeneous, orthotropic, and linear. The strain is found from the deformation of the beam, assuming that the perpendicular displacement is greater than the beam thickness, as shown in Fig. 1.

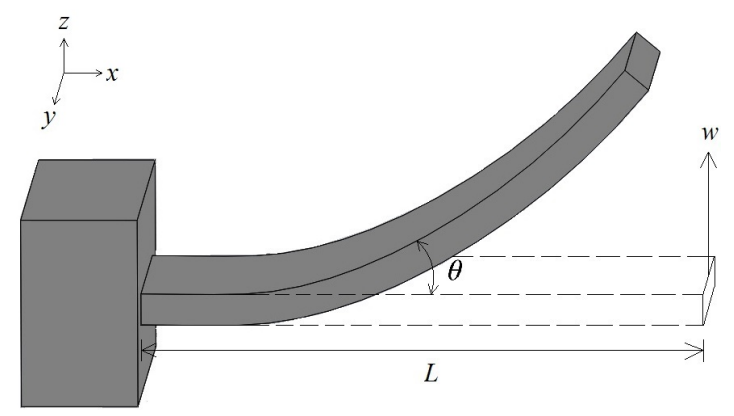

Fig. 1. Geometry of the cantilever beam

If the beam is represented as a one-dimensional element, and the strain only occurs along the $x$ direction; then, the strain field as a function of the bending curvature can be expressed as:

$$
\varepsilon=\frac{z\left(\frac{\partial^{2} w}{\partial x^{2}}\right)}{\sqrt{\left(1+\left(\frac{\partial w}{\partial x}\right)^{2}\right)^{3}}},
$$

where $z$ is the local coordinate perpendicular to the beam dimension, $x$ is the coordinate along the beam dimension and $w$ is the displacement along $z$. The subindex indicates partial derivatives with respect to $x$. Expanding the rational term as a convergent series:

$$
\frac{1}{\sqrt[2]{(1+m)^{3}}}=1-\frac{3}{2} m+\frac{15}{8} m^{2}-\frac{105}{48} m^{3}+\ldots
$$

Then, the strain function can be rearranged as:

$$
\varepsilon=z\left(\frac{\partial^{2} w}{\partial x^{2}}\right)\left(1-\frac{3}{2}\left(\frac{\partial w}{\partial x}\right)^{2}\right) .
$$

Using the Galerkin method, the displacement field can be approximated by a polynomial of the form:

$$
w=\sum_{i=1}^{N} a_{i} x^{i-1},
$$

where $N$ is the number of degrees of freedom in the element and the coefficients $a_{i}$ are determined from the generalized coordinates. If the beam is represented as a one-dimension element with two nodes, $j=1,2$, then, there are four generalized coordinates:

$$
\mathbf{u}=\left\{w_{1}, \theta_{1}, w_{2}, \theta_{2}\right\},
$$

where $\mathbf{u}$ is the generalized coordinates vector, $w_{j}$ is the displacement and $\theta_{j}$ are the bending angle of the corresponding nodes. Therefore, for this particular case, the polynomial in Eq. (4) has four coefficients.

The equation of motion is found using Lagrange's equation. The potential energy $V$ is determined from the strain energy function. Since it is a unidimensional element, it can be simplified as:

$$
V=\frac{1}{2} \int_{v} \sigma \varepsilon d v
$$

Considering only elements with linear material properties, the constitutive equation can be expressed as:

$$
\sigma=E \varepsilon
$$

Substituting Eq. (7) into Eq. (6), the potential energy is given as follow:

$$
V=\frac{1}{2} \int_{v} E \varepsilon^{2} d v
$$

In contrast, the kinetic energy is determined as:

$$
T=\frac{1}{2} \int_{v} \rho\left(\frac{d \mathbf{u}}{d t}\right)^{T}\left(\frac{d \mathbf{u}}{d t}\right) d v .
$$

Considering that the beam is homogeneous, and the cross section is constant, Eqs. (8) and (9) are included into Lagrange's equation. Then, four equations are derived, one for each generalized coordinate:

$$
m_{i} \frac{d^{2} u_{i}}{d t^{2}}+\sum_{k=1}^{4} \frac{\partial V}{\partial a_{k}} \frac{\partial a_{k}}{\partial u_{i}}=0,
$$

where $m_{i}$ is the associated mass to each node. Considering that the beam element is fixed at one end $w_{1}=0, \theta_{1}=0$ and redefining the other two generalized coordinates as $w_{2}=0, \theta_{2}=0$, Eq. (10) is reduced to two nonlinear equations:

$$
\begin{aligned}
& m \frac{d^{2} w}{d t^{2}}+\xi \frac{d w}{d t}+\frac{17496}{385} \frac{E I w^{5}}{L^{7}}-\frac{486}{11} \frac{E I \theta w^{4}}{L^{6}}+ \\
& +\left(\frac{1620}{11} \theta^{2}-\frac{1296}{5}\right) \frac{E I w^{3}}{7 L^{5}}-\left(\frac{729}{22} \theta^{3}-162\right) \frac{E I w^{2}}{7 L^{4}}+ \\
& +\left(\frac{54}{77} \theta^{4}-\frac{216}{35} \theta^{2}+12\right) \frac{E I w}{L^{3}}- \\
& -\left(\frac{522}{385} \theta^{4}-\frac{24}{7} \theta^{2}+6\right) \frac{E I \theta}{L^{2}}=F(t),
\end{aligned}
$$


$J \frac{d^{2} \theta}{d t^{2}}+\xi \frac{d \theta}{d t}-\frac{486}{55} \frac{E I w^{5}}{L^{6}}-\frac{810}{77} \frac{E I \theta w^{4}}{L^{5}}+$

$+\left(-\frac{729}{22} \theta^{2}+54\right) \frac{E I w^{3}}{7 L^{4}}+\left(\frac{108}{11} \theta^{2}-\frac{216}{5}\right) \frac{E I w^{2}}{7 L^{3}}+$

$+\left(-\frac{522}{77} \theta^{4}+\frac{72}{7} \theta^{2}-6\right) \frac{E I w}{L^{2}}+$

$+\left(\frac{1944}{385} \theta^{4}-\frac{256}{35} \theta^{2}+4\right) \frac{E I \theta}{L}=0$,

where $F(t)$ is the excitation force and the polynomial terms included in the stiffness are related to the restoring force generated by the potential energy. Therefore, the restoring force is modelled as a fifthdegree polynomial. In order to demonstrate the effectiveness of the model, experimental tests are also carried out. Then, both numerical and experimental data are analysed using different techniques.

\section{ANALYSIS TECHNIQUES}

Three different techniques are used to analyse numerical and experimental data: the frequency spectrum, the time-frequency map, and the phase diagram or phase plane [21].

The frequency spectrum is one of the most commonly used techniques for vibration analysis and machine-health monitoring. It is generated via the fast Fourier transform, and it provided the frequency content of the time-domain signal, showing up the harmonics frequency content. However, by itself, the frequency spectrum does not enable the analysis of the system evolution over time. The time-frequency map (spectrogram) is constructed using the continuous wavelet transform (CWT). The definition of the CWT is out of the scope of this work; readers are referred to [22]. The advantage of the CWT is that it can decompose a signal into a set of vectors within a set of frequencies. This set of frequencies displays the behaviour of each frequency as a function of time. If the system is linear, each frequency remains constant at any time; otherwise, it varies and, in the timefrequency map, it shows changes in the amplitude. The phase diagram, or phase plane, represents the instantaneous energy space. The vertical axis corresponds to the kinetic energy and the horizontal axis to the potential energy. If the dynamic response is stable, the trajectory described by the energy space will be a smooth function. Otherwise, the trajectory will display jumps or changes in direction, as well as many internal loops. Combining both analyses, phase diagram and time-frequency map, it is possible to determine the nonlinear behaviour of the cantilever beam.

\section{NUMERICAL SIMULATION}

Eqs. (11) and (12) were solved numerically. The solution was found using the Runge-Kutta method, assuming a sinusoidal excitation. Table 1 lists the beam parameters used in the model.

Table 1. Dimensions and properties of the modelling beam

\begin{tabular}{lc}
\hline Property & Value \\
\hline Width, $W$ & $0.025[\mathrm{~m}]$ \\
\hline Thickness, $T$ & $0.003[\mathrm{~m}]$ \\
\hline Length, $L$ & $1.000[\mathrm{~m}]$ \\
\hline Young's module, $E$ & $210[\mathrm{GPa}]$ \\
\hline Natural frequency, $\omega_{n}$ & $1.460[\mathrm{~Hz}]$ \\
\hline Mass, $m$ & $2.750[\mathrm{~kg}]$ \\
\hline Damping coefficient, $\xi$ & $0.269[\mathrm{~kg} / \mathrm{s}]$ \\
\hline
\end{tabular}

The numerical solution of the model was done considering different conditions: free vibration and forced vibration. The results and discussion of the numerical analysis are presented in the following subsections.

\subsection{Results of the Free Vibration Response}

First, the model was solved considering a small displacement in the free end of the beam as an initial condition. The results are shown in Fig. 2. The frequency spectrum shows two peaks at $1.49 \mathrm{~Hz}$ and $5.49 \mathrm{~Hz}$, which correspond to the first and second vibration modes, respectively. The linear behaviour of the beam can be seen more clearly in the timefrequency map, since the amplitude of each frequency remains constant at any time. Moreover, there are not vertical stripes connecting the frequencies content in the map. The phase diagram (Fig. 2) shows closed loops forming an annular shape; it verifies the stable response of the beam when a small displacement value is given.

In a second case, the model was solved given a large displacement on the free end of the beam as an initial condition. The results are shown in Fig. 3. The frequency spectrum displays two main peaks at 1.3 $\mathrm{Hz}$ and $5.4 \mathrm{~Hz}$. The first mode is shifted due to the nonlinear behavior, and both modes show sidebands at $2.4 \mathrm{~Hz}$ and $6.7 \mathrm{~Hz}$, respectively. The nonlinear behaviour is evident in the time-frequency map as the amplitudes vary over time. The phase diagram shows closed loops with two attracting poles, and they 

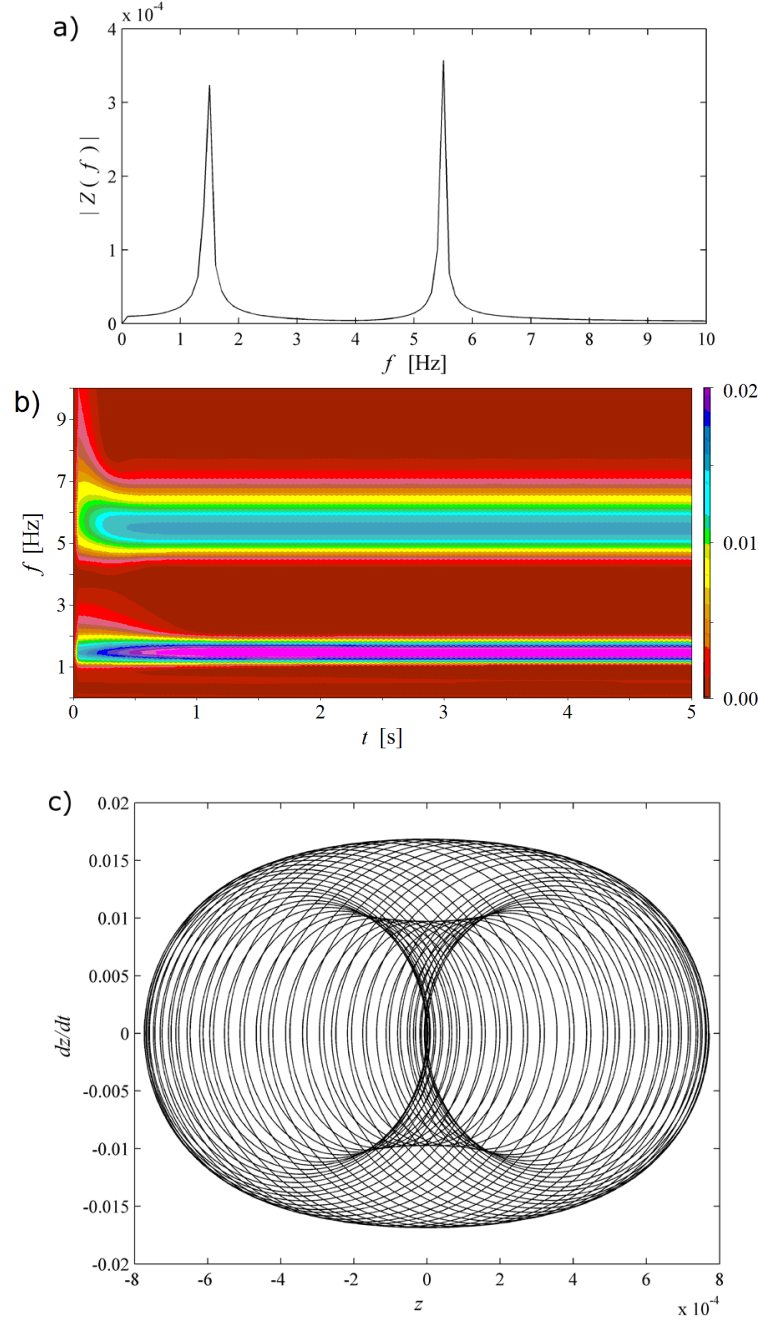

Fig. 2. Numerical response, free vibration response for small deformations; a) frequency spectrum; b) time-frequency map; c) phase diagram*

remain at the same location at any time. Comparing this diagram with the linear solution, the nonlinear effect is noted.

\subsection{Results of the Forced Vibration Response}

A forced vibration solution was made at the nonlinear frequencies found in the experimental test for free vibration analysis. Fig. 4 shows the results when the system is excited at $2.9 \mathrm{~Hz}$. The frequency spectrum shows a dominant peak at $2 \mathrm{~Hz}$ and a second and third ones at $5.9 \mathrm{~Hz}$ and $4.2 \mathrm{~Hz}$, respectively. In the timefrequency map, it can be seen that the frequencies have a nonlinear behaviour since they vary in time.
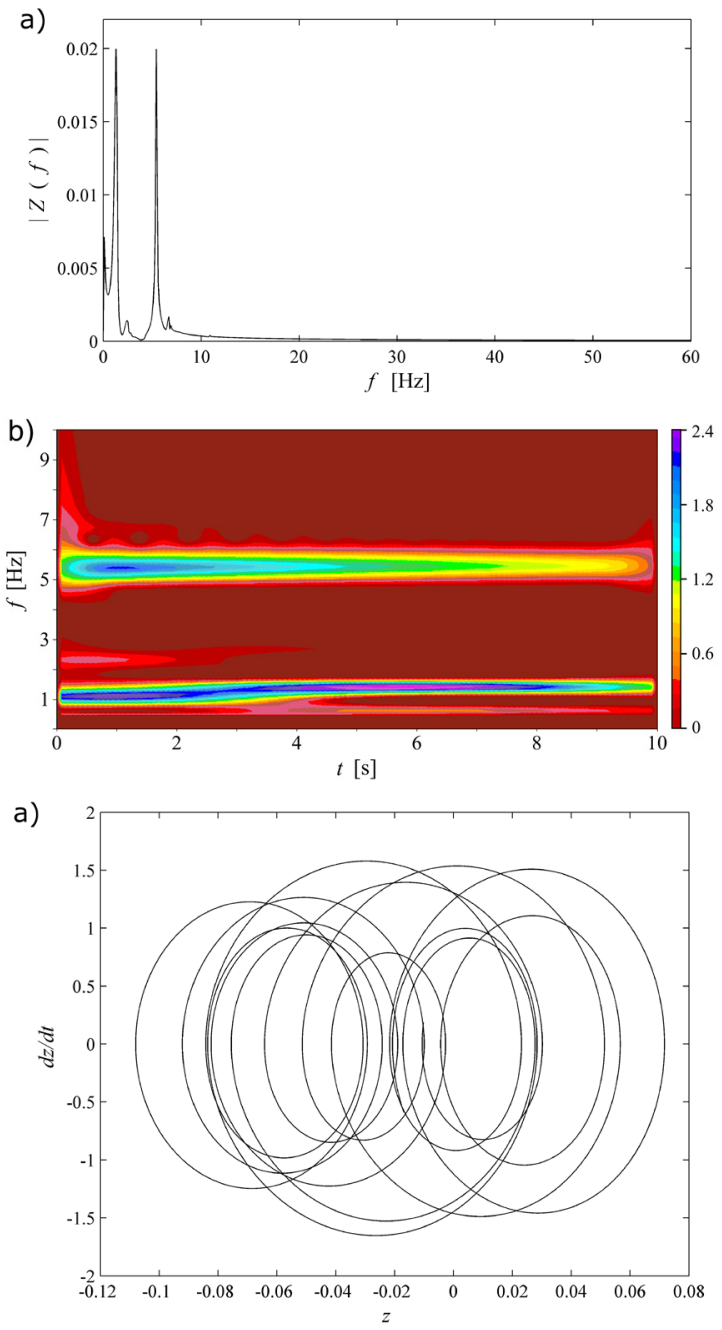

Fig. 3. Numerical response, free vibration response at large deformations; a) frequency spectrum; b) time-frequency map; c) phase diagram*

The phase diagram shows two attracting poles in the loops, also indicating the nonlinear behaviour.

Fig. 5 shows the results for the forced vibration at $18.1 \mathrm{~Hz}$. The frequency spectrum shows two main peaks: the dominant peak at the excitation frequency and the second one at $1.8 \mathrm{~Hz}$. The time-frequency map shows nonlinear behaviour at the excitation frequency. The phase diagram shows closed loops and unstable behaviour of the system.

Fig. 6 presents the results for the forced vibration at $51 \mathrm{~Hz}$. The frequency spectrum shows the dominant peak at the excitation frequency and the second peak at $1.4 \mathrm{~Hz}$. The time-frequency map displays the nonlinear behaviour near the $1.4 \mathrm{~Hz}$ frequency, since

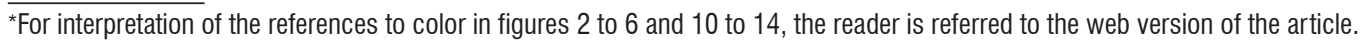


a)
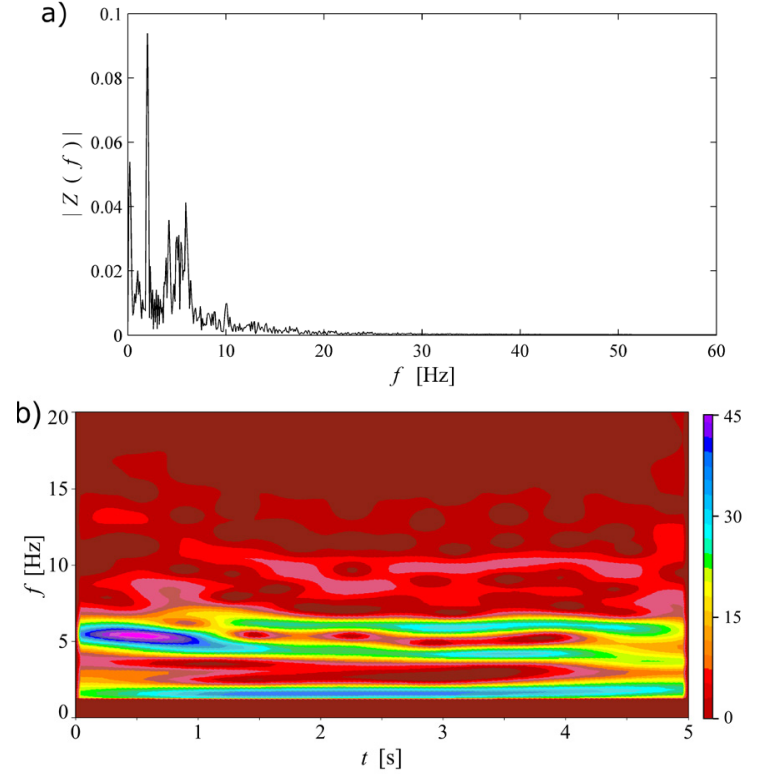

c)

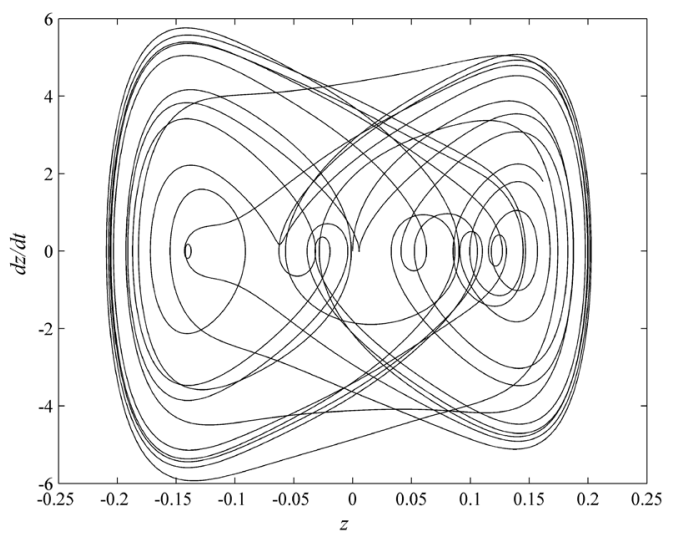

Fig. 4. Numerical response at $2.9 \mathrm{~Hz}$ excitation frequency; a) frequency spectrum; b) time-frequency map; c) phase diagram*

it diminishes in time. The phase diagram shows closed loops, which are shifted along the $x$-axis.

In order to validate the simplified model, a set of tests was conducted.

\section{EXPERIMENTAL VALIDATION}

Two kinds of tests were done on a slender cantilever beam: free vibration and forced vibration. The experimental test rig is shown in Fig. 7. Essentially, it consists of a cantilever beam (1), whose parameters are listed in Table 1; an ADX-321J accelerometer (2) placed at the free end of the beam to measure the vibration on the axis $z$ (see Fig. 1) (because the accelerometer mass is relatively small compared to the beam mass, it has not influence on the dynamic response of the beam); a data acquisition system NI
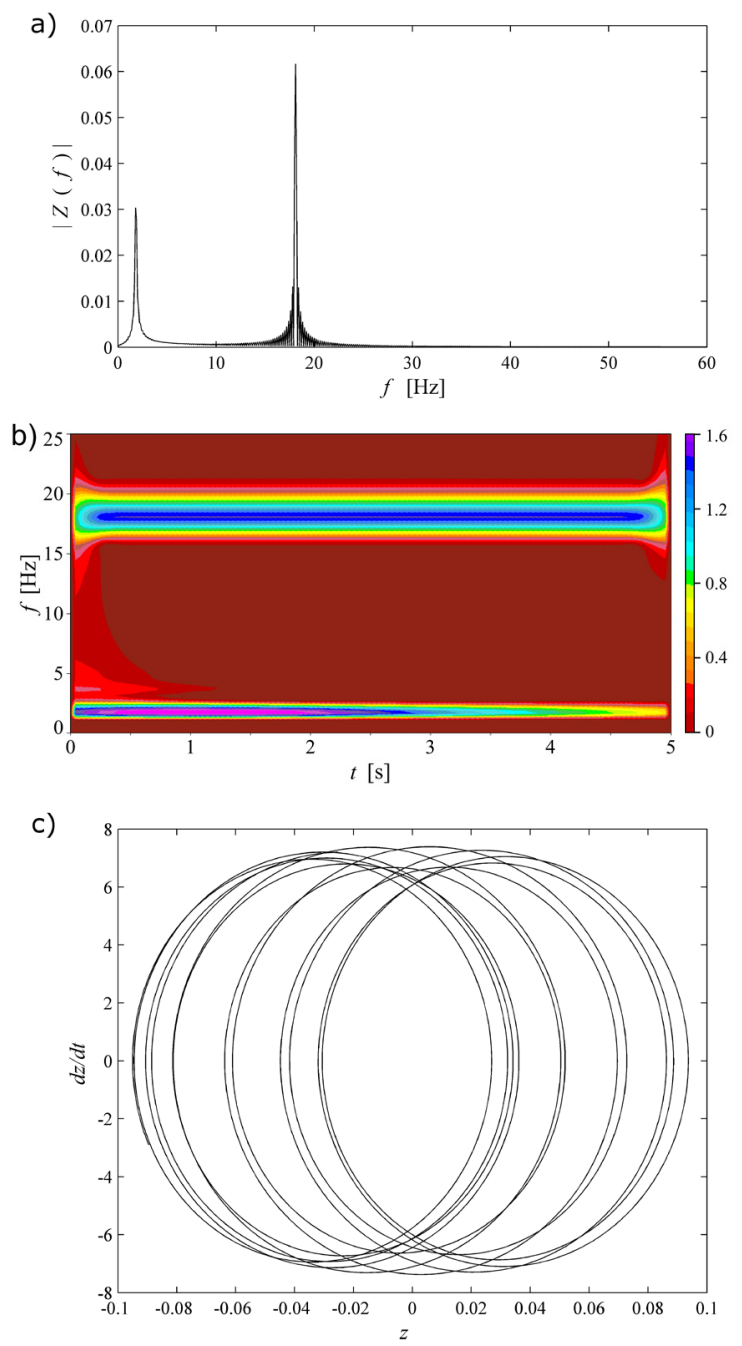

Fig. 5. Numerical response at $18.1 \mathrm{~Hz}$ excitation frequency; a) frequency spectrum; b) time-frequency map; c) phase diagram *

USB-6366 (3); and a PC (4). The data acquisition was executed from a graphical interface developed in LabView $^{\circledR}$. During the forced vibration test, the cantilever beam was excited by the TIRA ${ }^{\circledR}$ TV 51144IN inertial shaker (5). For this purpose, a frequency sweep of a sine vibration profile was generated by the Tektronix AFG 3102 signal generator (6); and then, the signal was amplified by the TIRA ${ }^{\circledR}$ BAA 1000 power amplifier (7) and then it was sent to the shaker to generate a sine oscillation. A schematic diagram of the test rig is shown in Fig. 8.

In order to produce the phase diagram, the displacement and velocity data were calculated numerically from the acceleration measurements. 
a)
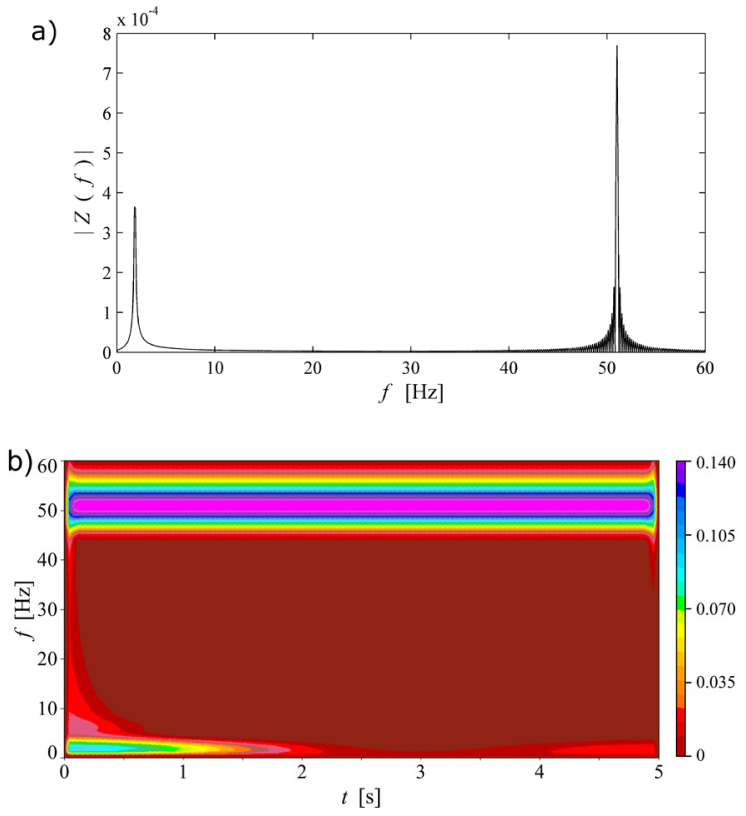

c)

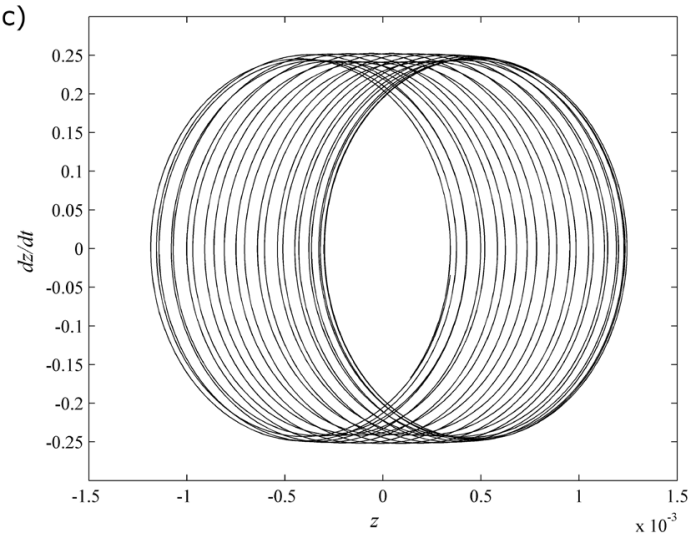

Fig. 6. Numerical response at $51 \mathrm{~Hz}$ excitation frequency; a) frequency spectrum; b) time-frequency map; c) phase diagram*

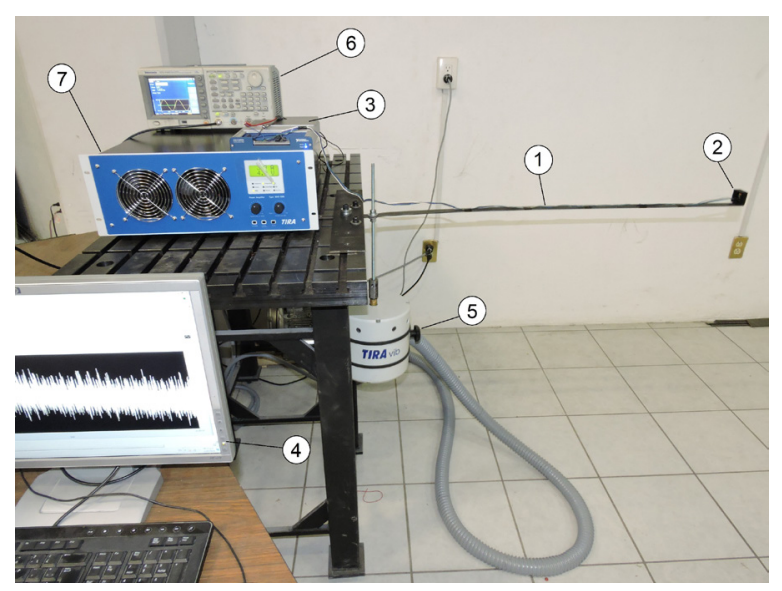

Fig. 7. Experimental test rig

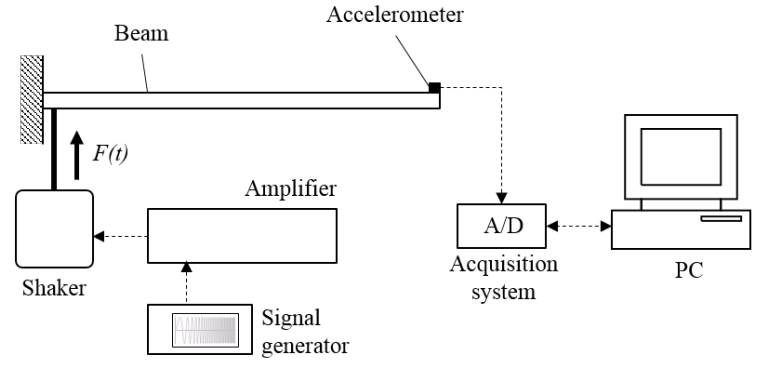

Fig. 8. Schematic diagram of the experimental test rig

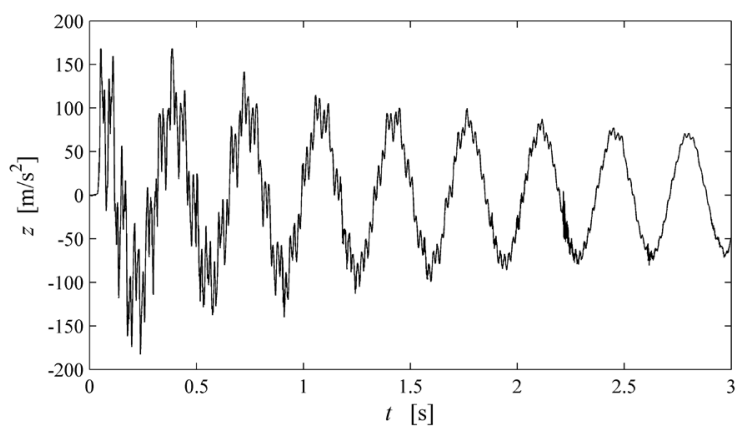

Fig. 9. Time response of the system for the free vibration test

\subsection{Results of the Free Vibration Response}

The time response for the free vibration test is shown in Fig. 9. In this figure, it is possible to see the nonlinear effect from the higher frequencies in the signal when the oscillations amplitude is higher; as the oscillations amplitude decreases, the system response is more linear.

The analysis of the free vibration response is presented in Fig. 10. The frequency spectrum shows a dominant peak at $2.9 \mathrm{~Hz}$, a second one around 18.01 $\mathrm{Hz}$, and a third one at $50.96 \mathrm{~Hz}$. The time-frequency map shows the damping effect, but the nonlinear behaviour is clearly identified around the $18.01 \mathrm{~Hz}$ and $50.96 \mathrm{~Hz}$ frequencies, as the frequency amplitude varies over time. The phase diagram shows a shape with a squared form at the outsides, caused by the nonlinear behaviour. The attracting poles diminished because the numerical integration filters the high nonlinear frequencies. Although the overall shape keeps a polygonal shape, the time-frequency map reveals the nonlinear behaviour at the second and third frequencies.

\subsection{Results of the Frequency Sweep Response}

A frequency sweep ramped up and down was applied to the beam while the vibration response was recorded. 
a)
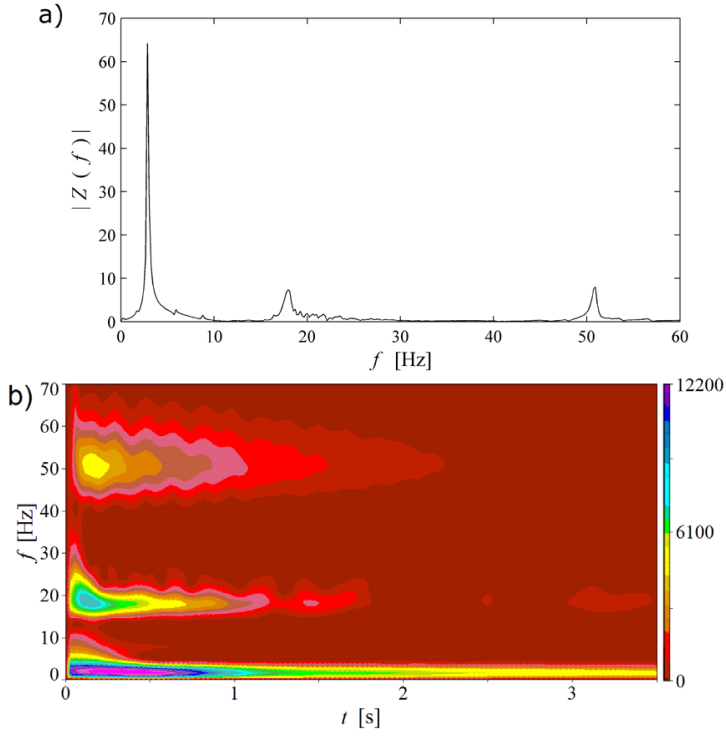

c)

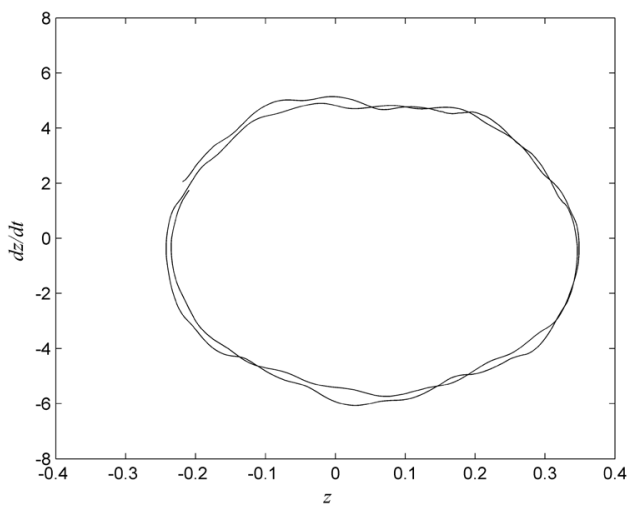

Fig. 10. Experimental results, free vibration; a) time response; b) frequency spectrum; c) time-frequency map; d) phase diagram*
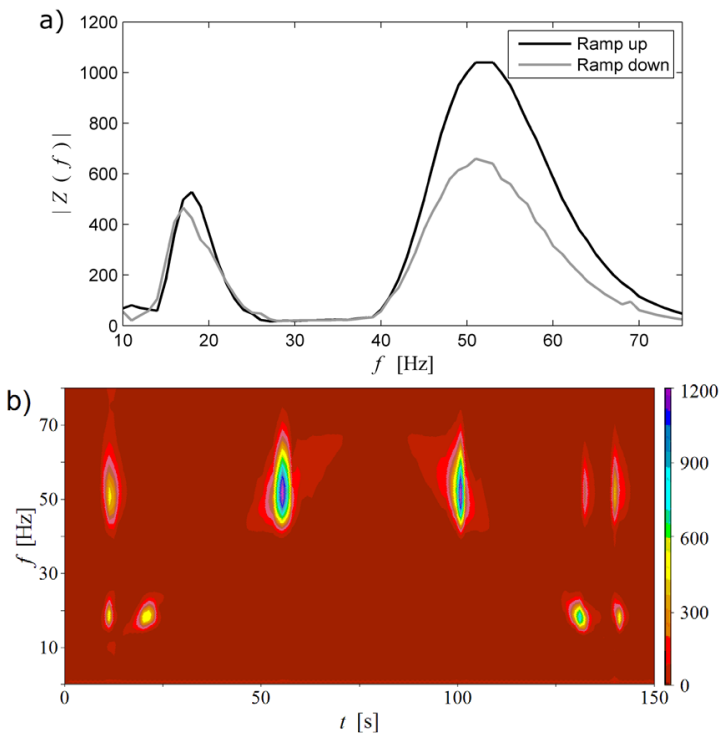

Fig. 11. Experimental frequency response obtained by frequency sweep; a) response amplitude; b) time-frequency map*
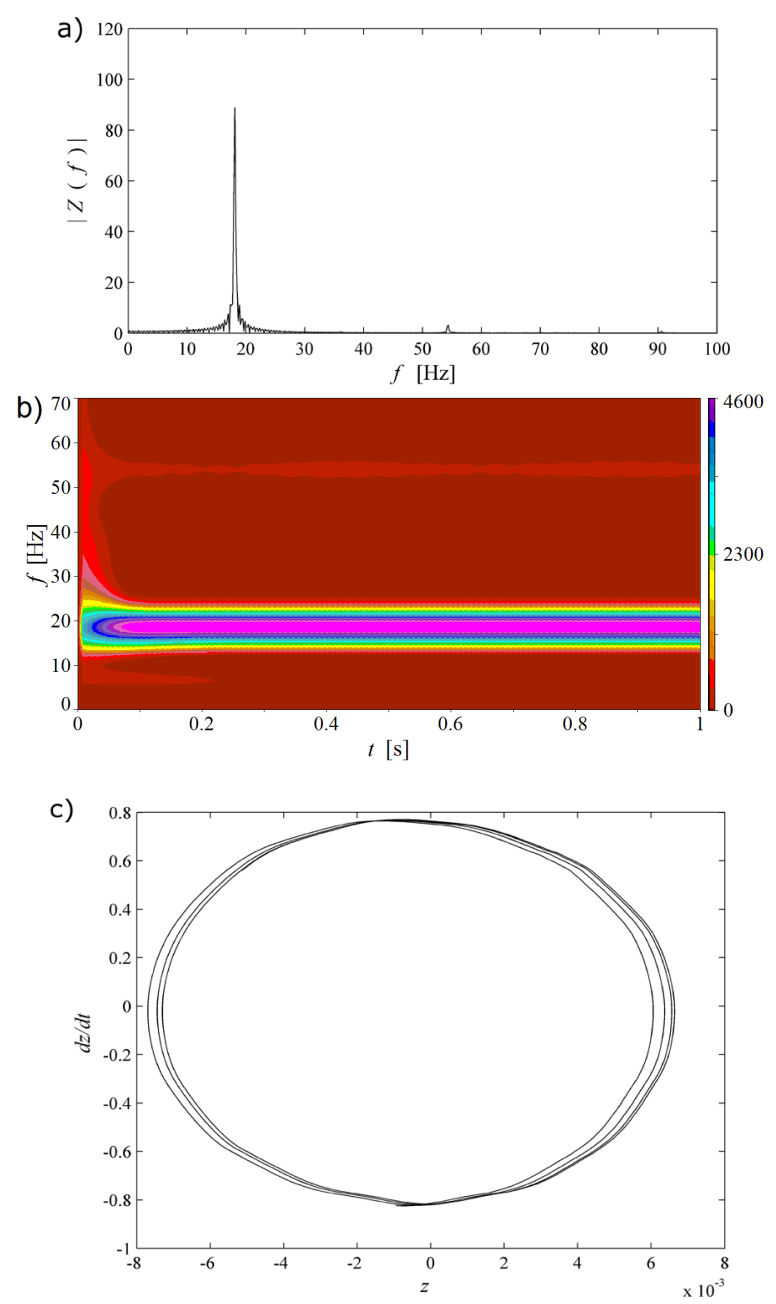

Fig. 12. Experimental results, Forced vibration at $18.1 \mathrm{~Hz}$; a) frequency spectrum; b) time-frequency map; c) phase diagram ${ }^{\star}$

The response amplitude is presented in Fig. 11a. It is clear that there is a jump phenomenon associated with the nonlinearities of the beam at the $18.1 \mathrm{~Hz}$ and 51 $\mathrm{Hz}$ frequencies. In the case of the excitation frequency at $51 \mathrm{~Hz}$, the beam shows relatively larger amplitudes. Therefore, the nonlinear behaviour is more noticeable at this frequency. The evolution in time of the system response is presented in Fig. 11b. It can be seen that the $51 \mathrm{~Hz}$ frequency is also excited when low frequencies are applied to the system.

Two forced vibration tests were conducted at the critical frequencies found in the jump diagram. The first measurement was recorded at an excitation frequency of $18.1 \mathrm{~Hz}$. The analysis results are presented in Fig. 12. The frequency spectrum shows a dominant peak at the excitation frequency and a second small peak at $54 \mathrm{~Hz}$. In the time-frequency 


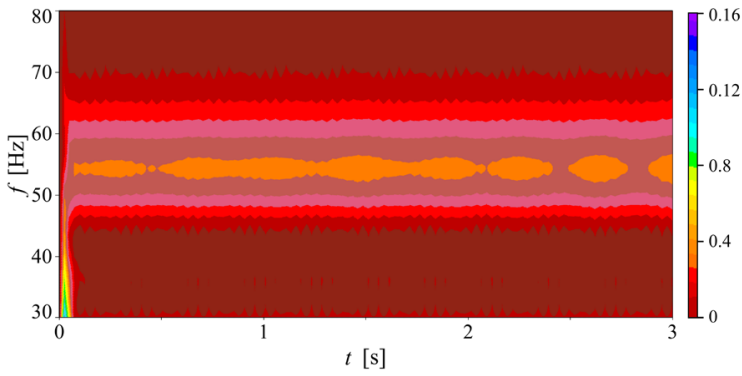

Fig. 13. Nonlinear response of the $54 \mathrm{~Hz}$ frequency when the beam is excited at $18.1 \mathrm{~Hz}^{*}$
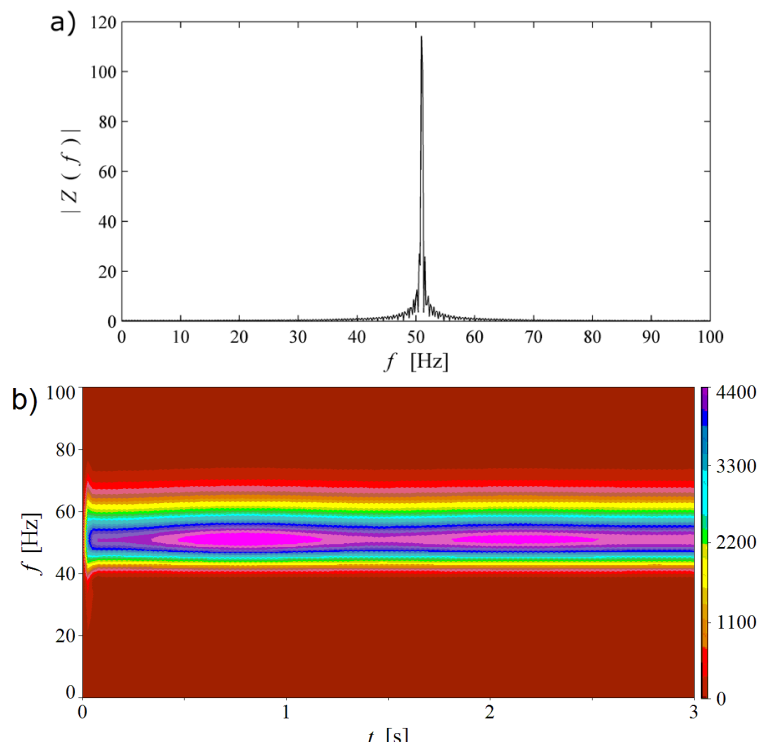

c)

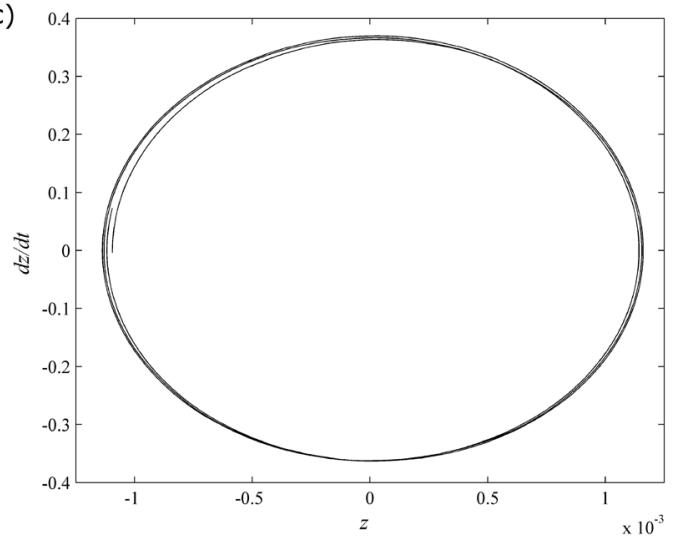

Fig. 14. Experimental results, forced vibration at $51 \mathrm{~Hz}$; a) frequency spectrum; b) time-frequency map; c) phase diagram*

map, it can be seen that the excitation frequency affects the system response. Measurements are dominated by the excitation frequency shadowing the beam response except at the other frequencies. Therefore, large amplitudes produced by the excitation frequency excites the nonlinear behaviour of the beam around $54 \mathrm{~Hz}$. Fig. 13 shows a close view of the nonlinear behaviour around $54 \mathrm{~Hz}$. The phase diagram shows closed loops with a balloon shape.

The second forced test was recorded at $51 \mathrm{~Hz}$. The analysis results at this condition are shown in Fig. 14. The frequency spectrum shows only a dominant peak around the excitation frequency, whereas the time-frequency map exhibits the nonlinear behavior at the excitation frequency. The phase diagram exhibits shifting closed loops. It is important to notice that the beam has a characteristic behaviour due to the large deformation effect. This behaviour occurs at a particular frequency that cannot be determined with the linear theory.

Large deformations are a source of nonlinearity in cantilever beams. The effect produces a dynamic response that occurs at a different frequency. This frequency has no relation to those associated with the linear modes of vibration. Additionally, the nonlinear effect due to large deformations only occurs when the displacement is significantly large. In such cases, it is necessary to displace the tip of beam several times its thickness or excite the beam with a frequency near the nonlinear frequency. However, in engineering problems these conditions are not easily found.

\section{CONCLUSIONS}

Cantilever beams can have a nonlinear dynamic response due to large deformations. This behaviour has been identified experimentally, and it can be simulated assuming the dynamic model has polynomial terms. The numerical solution shows a typical harmonic distortion pattern due to polynomial terms in the model. When the excitation frequency is below the natural frequency, the phase diagram has two attracting poles. Experimental results show two particular frequencies around $18 \mathrm{~Hz}$ and $51 \mathrm{~Hz}$, which are related to the nonlinear terms of the beam. Thus, these frequencies are associated with the large deformations effect. In the case of the excitation frequency at $51 \mathrm{~Hz}$, the beam shows highly different amplitudes. From the frequency sweep test, it is found that the amplitudes were higher in the ramp-up process than in the ramp down procedure. Therefore, the nonlinear behaviour is more noticeable at this frequency. Nevertheless, these frequencies can only be found numerically or experimentally. The nonlinear behaviour due to large deformations occurs only at certain frequencies or when the beam is deflected a large amount. Otherwise, the large deformation effects 
can be neglected. The simplified approximation presented in this work is sufficient for determining those frequencies that are due to the nonlinear terms. Any other method can produce these values only if the relative displacement between two points is significantly large.

\section{ACKNOWLEDGEMENTS}

The first author is grateful for the financial support from the Research Grants of the National Council for Science and Technology (CONACYT) in Mexico (No. Grant 238141).

\section{REFERENCES}

[1] Halilovič, M., Štok, B. (2007). Analytical tracing of the evolution of the elasto-plastic state during the bending of beams with a rectangular cross-section. Strojniški vestnik - Journal of Mechanical Engineering, vol. 53, no. 12, p. 806-818.

[2] Yu, A., Yang, R., Hao, Y. (2009). Theory and application of naturally curved and twisted beams with closed thin-walled cross sections. Strojniški vestnik - Journal of Mechanical engineering, vol. 55, no. 12, p. 733-741.

[3] Gerstmayr, J., Irschik, H. (2008). On the correct representation of bending and axial deformation in the absolute nodal coordinate formulation with an elastic line approach. Journal of Sound and Vibration, vol. 318, no. 3, p. 461-487, D0I:10.1016/J.jsv.2008.04.019.

[4] Ashour, O.N., Nayfeh, A.H. (2003). Experimental and numerical analysis of a nonlinear vibration absorber for the control of plate vibrations. Journal of Vibration and Control, vol. 9, no. 1-2, p. 209-234, D0l:10.1177/107754603030748.

[5] Bureau, E., Schilder, F., Santos, I.F., Thomsen, J.J., Starke, J. (2013). Experimental bifurcation analysis of an impact oscillator-tuning a non-invasive control scheme. Journal of Sound and Vibration, vol. 332, no. 22, p. 5883-5897, D0I:10.1016/j.jsv.2013.05.033.

[6] Malatkar, P., Nayfeh, A.H. (2003). A parametric identification technique for single-degree-of-freedom weakly nonlinear systems with cubic nonlinearities. Journal of Vibration and Control, vol. 9, no. 3-4, p. 317-336, DOI:10.1177/107754603030754.

[7] Paak, M., Païdoussis, M.P., Misra, A.K. (2013). Nonlinear dynamics and stability of cantilevered circular cylindrical shells conveying fluid. Journal of Sound and Vibration, vol. 332, no. 14, p. 3474-3498, D0l:10.1016/j.jsv.2013.01.030.

[8] Stoykov, S., Ribeiro, P. (2010). Nonlinear forced vibrations and static deformations of $3 D$ beams with rectangular cross section: the influence of warping, shear deformation and Iongitudinal displacements. International Journal of Mechanical Sciences, vol. 52, no. 11, p. 1505-1521, D0I:10.1016/j.jijmecsci.2010.06.011.

[9] Li, L., Zhang, D. (2015). Dynamic analysis of rotating axially FG tapered beams based on a new rigid-flexible coupled dynamic model using the B-spline method. Composite Structures, vol. 124, p. 357-367, D0l:10.1016/j.compstruct.2015.01.018.

[10] Stangl, M., Gerstmayr, J., Irschik, H. (2008). An alternative approach for the analysis of nonlinear vibrations of pipes conveying fluid. Journal of Sound and Vibration, vol. 310, no. 3, p. 493-511, D0l:10.1016/j.jsv.2007.06.020.

[11] Wang, J.F., Liew, K.M. (2015). An accurate improved complex variable element-free method for numerical solutions of elastodynamic problems. Engineering Analysis with Boundary Elements, vol. 50, p. 304-312, D0l:10.1016/j. enganabound.2014.09.005.

[12] Wang, Y.-G., Lin, W.-H., Liu, N. (2013). Nonlinear free vibration of a microscale beam based on modified couple stress theory. Physica E: Low-dimensional Systems and Nanostructures, vol. 47, p. 80-85, D0l:10.1016/j.physe.2012.10.020.

[13] Zhang, W., Sun, L., Yang, X.D., Jia, P. (2013). Nonlinear dynamic behaviors of a deploying-and-retreating wing with varying velocity. Journal of Sound and Vibration, vol. 332, no. 25, p. 6785-6797, D0l:10.1016/j.physe.2012.10.020.

[14] Noor, A.K., Peters, J.M., Min, B.-J. (1989). Mixed finite element models for free vibrations of thin-walled beams. Finite Elements in Analysis and Design, vol. 5, no. 4, p. 291-305, D0I:10.1016/0168-874X(89)90009-7.

[15] Machado, S.P., Cortínez, V.H. (2004). Nonlinear geometric analysis of composite thin-walled beams. Mecánica Computacional, vol. 23, no. 5, p. 391-410.

[16] Ramezani, S. (2012). A micro scale geometrically non-linear Timoshenko beam model based on strain gradient elasticity theory. International Journal of Non-Linear Mechanics, vol. 47, no. 8, p. 863-873, D0l:10.1016/j.jjnonlinmec.2012.05.003.

[17] Kang, Y.-A., Li, X.-F. (2009). Bending of functionally graded cantilever beam with power-law non-linearity subjected to an end force. International Journal of Non-Linear Mechanics, vol. 44, no. 6, p. 696-703, DOl:10.1016/j.ijnonlinmec.2009.02.016.

[18] Fotouhi, R. (2007). Dynamic analysis of very flexible beams. Journal of Sound and Vibration, vol. 305, no. 3, p. 521-533, DOI:10.1016/j.jsv.2007.01.032.

[19] Shad, M.R., Michon, G., Berlioz, A. (2011). Modeling and analysis of nonlinear rotordynamics due to higher order deformations in bending. Applied Mathematical Modelling, vol. 35, no. 5, p. 2145-2159, D0l:10.1016/J.apm.2010.11.043.

[20] Jauregui-Correa, J.C., Gonzalez Brambila, O.M. (2009). Mechanical Vibrations of Discontinuous Systems. Nova Science Publishers, New York.

[21] Jauregui, J.C. (2014). Parameter Identification and Monitoring of Mechanical Systems under Nonlinear Vibration. Elsevier, Cambridge.

[22] Gao, R.X., Yan, R. (2011). Wavelets, Theory and Applications for Manufacturing. Springer, New York. 